\title{
Four chamber right ventricular longitudinal strain versus right free wall longitudinal strain. Prognostic value in patients with left heart disease
}

Ana García-Martín ${ }^{1}$ José-Luis Moya-Mur ${ }^{1}$, Sonsoles Alejandra Carbonell-San Román Alberto García-Lledó ${ }^{1}$, Paula Navas-Tejedor ${ }^{2}$, Alfonso Muriel ${ }^{1}$, Daniel Rodríguez-Muñoz ${ }^{1}$, Eduardo Casas-Rojo ${ }^{1}$, Jose-Julio Jiménez-Nacher ${ }^{1}$, Covadonga Fernández-Golfín ${ }^{1}$, José-Luis Zamorano ${ }^{1}$

${ }^{1}$ Ramón y Cajal University Hospital, Madrid, Spain

${ }^{2}$ Gregorio Marañón University Hospital, Madrid, Spain

\begin{abstract}
Background: There is no consensus on which right ventricle (RV) strain parameter should be used in the clinical practice: four chamber RV longitudinal strain (4CH RV-LS) or free wall longitudinal strain (FWLS). The aim of this study was to analyze which RV strain parameter better predicts prognosis in patients with left heart disease.

Methods: One hundred and three outpatients with several degrees of functional tricuspid regurgitation severity secondary to left heart disease were prospectively included. 4CH RV-LS and FWLS were assessed using speckle tracking. Left ventricular (LV) systolic function was determined using $L V$ ejection fraction and $R V$ systolic function using tricuspid annular plane systolic excursion (TAPSE). Patients were followed up for $23.1 \pm 12.4$ months for an endpoint of cardiac hospitalization due to heart failure.

Results: The cutoff value related to $R V$ dysfunction (TAPSE $<17 \mathrm{~mm}$ ) was lower, in absolute

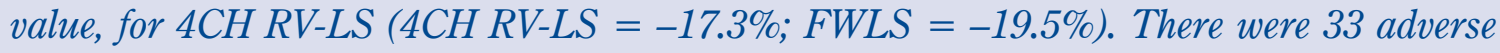
events during the follow-up. Patients with $4 C H R V-L S>-17.3 \%$ (log rank [LR] = 22.033; $p<0.001)$; FWLS > -19.5\% (LR = 12.2; $p<0.001)$, TAPSE < 17 mm (LR = 17.4; $p<0.001)$ and LV systolic dysfunction ( $L R=13.3 ; p<0.001)$ had lower event-free survival (Kaplan Meier). In Cox multivariate analysis, $4 C H R V-L S>-17.3 \%$ (hazard ratio [HR] = 3.593; $p<0.002)$, TAPSE $<17$ (HR = 2.093; $p<0.055)$ and LV systolic dysfunction $(H R=2.087$; $p<0,054)$ had prognostic value, whereas FWLS did not reach significance.
\end{abstract}

Conclusions: Although both $4 C H$ RV-LS and FWLS have prognostic value, 4CH RV-LS is a better predictor of episodes of heart failure in patients with left heart disease, providing additional information to that obtained by TAPSE. (Cardiol J 2016; 23, 2: 189-194)

Key words: right ventricle, right ventricular function, strain, echocardiography, heart failure

Address for correspondence: Ana García-Martín, MD, Department of Cardiology, Ramón y Cajal University Hospital, Ctra, Colmenar km 9,100, 28034 Madrid, Spain, tel: +34913368259, e-mail: aggarciamartin@gmail.com 


\section{Introduction}

Assessment of right ventricular (RV) function is of great importance for the management and prognosis of several heart diseases [1-3]. Its echocardiographic evaluation is limited mainly due to its complex anatomy and its high dependence on loading conditions.

Myocardial deformation techniques have been proposed as a different tool to evaluate RV function. Strain parameters provide a quantitative evaluation of global and regional deformation of the RV and speckle tracking assessment may grant certain advantages over Doppler tissue image such as angle-independence [4]. Due to the longitudinal alignment of deep muscle fibers of the RV from the base to the apex [5], longitudinal shortening is a major contribution of the RV function [6]; hence longitudinal strain can represent RV systolic function accurately. However, standardized data and consensus on which strain parameter is better related to RV function, whether four-chamber RV longitudinal strain (4CH RV-LS) or the single measurement of its free wall (FWLS), are lacking. The major difference is that $4 \mathrm{CH}$ RV-LS includes the interventricular septum which is an integral part of both ventricles. Some groups prefer FWLS, as they consider that the RV free wall contributes to the most important part in RV stroke volume, and to avoid overlapping with left ventricular (LV) global longitudinal strain [7], whereas others prefer $4 \mathrm{CH}$ RV-LS in order to assess the entire ventricle [3].

In this study, we sought to assess which RV strain parameter better correlates to prognosis in patients with left heart disease.

\section{Methods}

\section{Patient characteristics}

The study was carried out in a large tertiary care hospital. Out-patients with different types of left heart disease referred to our cardiac imaging unit were prospectively included.

In order to avoid potential selection bias choosing the patients in accordance with a given $\mathrm{RV}$ systolic function parameter, but at the same time trying to get a homogeneous representation of different degrees of RV dysfunction, we selected patients with different degrees of functional tricuspid regurgitation (TR), an indirect parameter of RV function. Thus, we included consecutive patients with different degree of TR severity associated to left heart disease.
Congenital heart disease, previous cardiac surgery, primary tricuspid disease and all types of pulmonary hypertension other than group 2 [8] were defined as exclusion criteria.

Patients' condition at the time of the study, including the presence of ankle swelling and the need for diuretic treatment was recorded. Patients were followed over $23.1 \pm 12.4$ months for an endpoint of cardiac hospitalization due to heart failure. Clinical events were obtained from medical records and direct interviews with patients. All work was in compliance with the declaration of Helsinki and performed with the approval of the local Ethics Committee.

\section{Echocardiography}

A complete transthoracic echocardiogram was performed using a Vivid E9 device (GE Medical Systems, Horten, Norway) according to the standard protocol $[9,10]$. All the studies were performed by two experienced echocardiographers (AG, JLM). Left atrial size was measured at its antero-posterior diameter from the long axis parasternal view in the end-systolic period and by its volume indexed volume from an apical 4-chamber view. LV ejection fraction (LVEF) was measured using apical biplane Simpson's method. LV dysfunction was defined as LVEF $<52 \%$ for men and LVEF $<54 \%$ for women according to the current recommendations [10]. The severity of TR was assessed using an integrated method including the estimation color area of the regurgitant jet in the right atrium, the vena contracta width (severe if the width of the vena contracta $\geq 7 \mathrm{~mm}$ ), the proximal isovelocity surface area (PISA) method with Nyquist limit shift of $28 \mathrm{~cm} / \mathrm{s}$ (mild if the radius of PISA was $\leq 5 \mathrm{~mm}$, severe if the radius of PISA was $\geq 9 \mathrm{~mm}$ ) and with the signal intensity and shape of the continuous wave Doppler of TR jet, according to the established practice guidelines [11]. TR was finally graded using a 4-point scale (normal, mild, moderate and severe). The systolic pulmonary artery pressure (SPAP) was estimated using TR peak velocity and right atrial pressure, which was estimated by the inferior vena cava diameter from long-axis subxiphoid view and its response to inspiration.

Two-dimensional speckle-tracking echocardiographic analysis was performed from a 4-chamber modified apical view focused on the RV. Three consecutive heart cycles were recorded and averaged. Images were optimized for strain analysis to improve frame rate to $50-90 \mathrm{frame} / \mathrm{s}$. The analysis was performed offline by using Echo-PAC software (GE Medical System, Horton, Norway). To calculate $4 \mathrm{CH}$ 


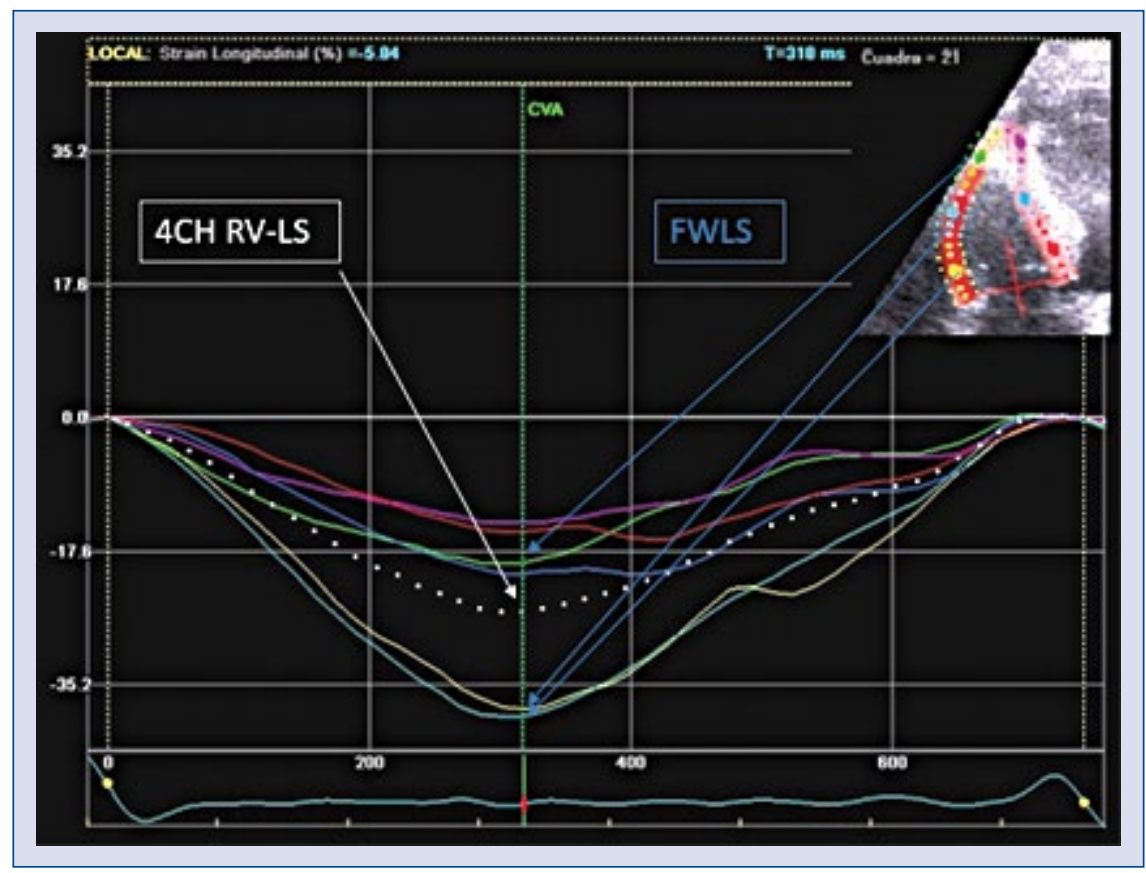

Figure 1. Right ventricular myocardial deformation study. Four chamber right ventricular longitudinal strain (4CH RV-LS) is obtained calculating the average peak strain values of the 6 analyzed segments. In the graphs, the dotted lines represent its value. Free wall longitudinal strain (FWLS) is obtained from the average of basal (yellow), medium (blue) and apical (green) values of right ventricle free wall.

RV-LS, the endocardial edge of the RV was manually traced in the 4-chamber view. The region of interest, composed from 6 segments, was automatically positioned to track frame by frame the RV throughout the cardiac cycle. The endocardial contour and width were manually adjusted when necessary to provide optimal tracking [12]. 4CH RV-LS was calculated as the mean of peak systolic values observed in the 6 segments, while FWLS was calculated as the mean of basal, mid and apical RV free-wall values (Fig. 1). Tricuspid annulal plane systolic excursion (TAPSE) was estimated by M-mode [mm], measured between end-diastole and peak-systole. RV dysfunction was defined as TAPSE $<17 \mathrm{~mm}$ according to the current recommendations [10].

\section{Statistical analysis}

Continuous variables are shown using mean and standard deviation; discrete variables using absolute and relative frequency. Chi-square test was used among discrete variables for statistical analysis. Fisher's test was used in cases where frequency was below 5. Assumption normality was performed by histogram and by KolmogorovSmirnov test. Student's t-test was used for comparison of continuous variables, or Mann-Whitney test when conditions of normal distribution were not met. For variables correlation contrast, Pearson correlation test was used, or Spearman's test when conditions of normal distribution were not met. The optimal cutoff value related to RV dysfunction defined by TAPSE $<17 \mathrm{~mm}$ was determined by the receiver-operating characteristic curve analysis. In two models of linear regression, $4 \mathrm{CH}$ RV-LS and FWLS were set as dependent variables, with LVEF, and TAPSE as co-variables, with a backwards strategy. Kaplan-Meier survival plots were calculated from baseline to time of adverse event and compared using the log-rank test. Cox proportional-hazards regression model was used to assess the risk of combined endpoint associated with $4 \mathrm{CH}$ RV-LS, FWLS, LVEF and TAPSE. Statistical analysis was performed using SPSS system version 16 and Stata 13. All p values reported are from 2 -sided tests, and $\mathrm{p}$ values $<0.05$ were considered statistically significant.

\section{Results}

\section{Patient characteristics}

A total 103 patients (67 females, mean age $72.9 \pm 14$ years old) with different left heart diseases were included in this study. Sixteen percent with severe TR, $30 \%$ with moderate TR, $29 \%$ with mild 
Table 1. Clinical and echocardiographic characteristics.

\begin{tabular}{|c|c|}
\hline Age [years] & $72.9 \pm 14.4$ \\
\hline Women & $65 \%$ \\
\hline Atrial fibrillation & $41 \%$ \\
\hline Hypertensive cardiomyopathy & $25 \%$ \\
\hline Ischemic cardiomyopathy & $17 \%$ \\
\hline Myocardial infarction & $11 \%$ \\
\hline Dilated cardiomyopathy & $8 \%(n=8)$ \\
\hline $\begin{array}{l}\text { Moderate to severe left heart } \\
\text { valvulopathy }\end{array}$ & $34 \%(n=35)$ \\
\hline $\begin{array}{l}\text { Aortic valve*: stenosis/ } \\
\text { /regurgitation }\end{array}$ & $12 \% / 9 \%$ \\
\hline $\begin{array}{l}\text { Mitral valve*: stenosis/ } \\
\text { /regurgitation }\end{array}$ & $10 \% / 19 \%$ \\
\hline LA dimension [mm] & $45.2 \pm 10.2$ \\
\hline Indexed LA volume $\left[\mathrm{cc} / \mathrm{m}^{2}\right]$ & $38 \pm 13$ \\
\hline LVEF [\%] & $64.3 \pm 13.9$ \\
\hline LV systolic dysfunction & $18 \%$ \\
\hline SPAP $[\mathrm{mm} \mathrm{Hg}]$ & $49.6 \pm 19.0$ \\
\hline $\begin{array}{l}\text { TRS (severe, moderate, mild, } \\
\text { none) }\end{array}$ & $16 \% / 30 \% / 29 \% / 25 \%$ \\
\hline TAPSE $[\mathrm{mm}]$ & $20.1 \pm 4.9$ \\
\hline RV systolic dysfunction & $31 \%(n=32)$ \\
\hline 4CH RV-LS & $-19.35 \pm 5.6$ \\
\hline FWLS & $-21.85 \pm 7.3$ \\
\hline RA pressure [mm Hg] & $9.4 \pm 5.8$ \\
\hline Previous HF & $38 \%$ \\
\hline Ankle swelling & $29 \%$ \\
\hline Diuretic treatment & $41 \%$ \\
\hline
\end{tabular}

Functional class $\geq 261.2 \%$ *Moderate or severe impairment; LA left atrium; LVEF — left ventricular ejection fraction; LV — left ventricle; SPAP - systolic pulmonary artery pressure; TRS - tricuspid tricle; SPAP - systolic pulmonary artery pressure; TRS - tricusp
regurgitation severity; TAPSE - tricuspid annular plane systolic excusion; RV - right ventricle; 4CH RV-LS - four chamber right ventricular longitudinal strain; FWLS - free wall longitudinal strain; $\mathrm{RA}$ - right atrium; HF — heart failure

TR and $25 \%$ without TR. LV systolic dysfunction was present in $18 \%$ of the total of the population and RV systolic dysfunction in $31 \%$. Sixty-one percent of patients were in functional class $\geq 2,38 \%$ had completed prior admission for heart failure and $41 \%$ needed diuretic therapy chronically. The clinical and echocardiographic data of the population are summarized in Table 1.

\section{Right ventricular strain and biventricular function}

4CH RV-LS and FWLS showed significant correlations with TAPSE $(r=-0.520, p<0.005$ and $\mathrm{r}=-0.471, \mathrm{p}<0.001$, respectively). The best cutoff value related to RV dysfunction (TAPSE <
$<17 \mathrm{~mm})$ was lower for of 4CH RV-LS $(-17.3 \%$; area under the curve $[\mathrm{AUC}]=0.775, \mathrm{p}<0.01$ ) than for FWLS $(-19.5 \%$; AUC $=0.754, \mathrm{p}<0.001)$.

Linear regression analysis showed FWLS depended exclusively on TAPSE (coefficient of determination 0.186; correlation with TAPSE $=-0.613$; 95\% confidence interval [CI]: $-0.865-0.360$ ), while 4CH RV-LS depended on LVEF and TAPSE (coefficient of determination 0.327 ; correlation with $\mathrm{LVEF}=-0.104$; 95\% CI: -0.174 to -0.033 ; correlation with TAPSE $=-0.492 ; 95 \%$ CI: $0.694-0.290$ ).

\section{Right ventricular strain and outcome}

During a follow-up of $23.1 \pm 12.4$ months, there were 33 adverse events. In Kaplan-Meier analysis, patients $4 \mathrm{CH}$ RV-LS $>-17.3 \%$ (log rank $[\mathrm{LR}]=22.033 ; \mathrm{p}<0.001)$, TAPSE $<17 \mathrm{~mm}$ $(\mathrm{LR}=17.4 ; \mathrm{p}<0.001), \mathrm{FWLS}>-19.5 \%(\mathrm{LR}=12.2$; $\mathrm{p}<0.001)$ and $\mathrm{LV}$ systolic dysfunction $(\mathrm{LR}=13.3$; $\mathrm{p}<0.001$ ) had lower event-free survival (Fig. 2).

After univariate analysis by Cox-proportional hazard regression analysis, TAPSE, LVEF, $4 \mathrm{CH}$ RV-LS and FWLS were associated with adverse outcomes (hazard ratio [HR] for a 4CH RV-LS $>-17.3 \%=5.19 ; \mathrm{p}<0.001 ;$ HR for a FWLS $>-19.5 \%=3.38 ; \mathrm{p}<0.001)$. In Cox multivariate analysis, with TAPSE $<17 \mathrm{~mm}, 4 \mathrm{CH}$ RV-LS $>-17.3 \%$, FWLS $>-22.7 \%$ and low LVEF; only 4CH RV-LS $>-17.3 \%(\mathrm{HR}=3.593 ; \mathrm{p}<0.002)$, TAPSE $<17(\mathrm{HR}=2.093 ; \mathrm{p}<0.055)$ and LV systolic dysfunction $(\mathrm{HR}=2.087 ; \mathrm{p}<0.054)$ had prognostic value whereas FWLS did not reach significance. The inclusion of the TR severity and the SPAP in the model did not change the results.

\section{Discussion}

In the present study, the authors assessed the prognostic value of $4 \mathrm{CH}$ RV-LS and FWLS in patients with left heart disease. The main finding is that although both parameters are prognostic markers, 4CH RV-LS shows a stronger association with episodes of heart failure than FWLS, providing additional prognostic information to that obtained with TAPSE and LVEF.

The fact that $4 \mathrm{CH}$ RV-LS has greater prognostic value than FWLS points to the importance of the septum in global RV function. In our study, both strain parameters correlate similarly with RV systolic function estimated by TAPSE, however, 4CH RV-LS has a stronger correlation with LVEF than FWLS suggesting that 4CH RV-LS is not only related with the RV function but also reflects the influence of $L V$ function on it. This parameter could 


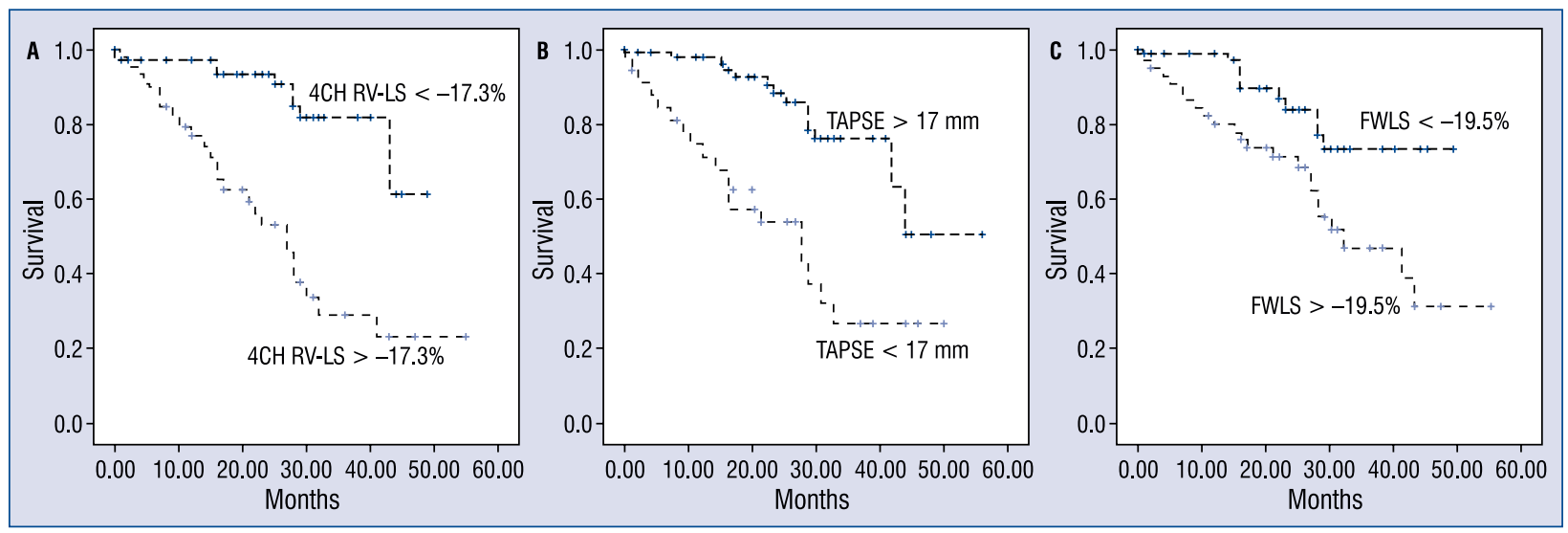

Figure 2. Kaplan-Meier analysis according to four chamber right ventricular longitudinal strain (A. 4CH RV-LS; > -17.3\%) and free wall longitudinal strain (C. FWLS; > $-19.5 \%$ ); B. Tricuspid annular plane systolic excursion (TAPSE).

therefore be considered a marker of biventricular function, a fact that could partly explain its better prognostic significance. Our finding is consistent with a general agreement that the malfunction of one chamber may influence the function of the neighboring chamber [13].

Recent publications have pointed to the prognostic value of RV strain in patients with heart failure $[3,14,15]$, but mostly in patients with severe LV dysfunction. Our data is consistent with that of Motoki et al. [3], who found that in patients with heart failure and low LVEF (25 $\pm 6 \%), 4 \mathrm{CH}$ RV-LS is a more reliable predictor of events than FWLS. The cutoff point shown in this study in event prediction (4CH RV-LS $>-14.8 \%$ ) is above ours $(-17.3 \%)$. Besides differences between vendors, their patients had severe LV dysfunction, thus tending to worse the value of RV longitudinal strain, as opposed to our population. Therefore, the $4 \mathrm{CH}$ RV-LS values used in patients with LV dysfunction are not fully extrapolated to other populations with normal LVEF. According to these data, $4 \mathrm{CH}$ RV-LS should be preferred over the FWLS when the objective is to assess the prognosis of patients with left heart disease. The FWLS could become a useful tool for the evaluation of isolated RV function, as in the study of right cardiomyopathy [16] or after interventionism as the impact of ventricular assistance devices [17].

Clinical practice guidelines for the assessment of the right heart [18] do not establish a strain cutoff point for RV systolic dysfunction evaluation. The latest recommendations for quantification of cardiac cavities [10] first suggested as abnormal a cutoff value for longitudinal RV FWLS strain of $>-20 \%$, while recognizing that the values may depend on the vendor and version of the used program. Meris et al. [12] established the cutoff value for $4 \mathrm{CH}$ RV-LS in $-19 \%$, Lee et al. [19] in $-16.8 \%$ and Park et at. [14] in $-15.4 \%$; for FWLS, Ternacle et al. [7] established the cutoff in $-21 \%$. In our sample of patients, using TAPSE as a reference $[18,20]$, the best value related to RV systolic dysfunction for 4CH RV-LS (-17.3\%) was lower in absolute value than for FWLS (-19.5\%).

We have to take into account that our sample was selected by its TR severity. It is difficult to assess the influence that the load conditions may have on the deformation and the strain results. The intrinsic RV function may be somewhere hidden in the dataset. Therefore, different load conditions and LV function probably impact on RV deformation and may partially explain the different values published in the literature.

Nevertheless, our data reflect that although both RV strain parameters reflects in somehow RV function, the cutoff values related to RV dysfunction should be lower in absolute value for $4 \mathrm{CH}$ RV-LS.

\section{Limitations of the study}

As a limitation of this study, it must be pointed out that data on RV ejection fraction as an indicator of the RV systolic function are missing. Although previous studies have validated the $4 \mathrm{CH}$ RV-LS with RV ejection fraction by cardiac magnetic resonance [14] and although the RV ejection fraction obtained by echocardiography also has limitations, its estimation would have offered interesting information to the study.

To avoid a selection bias we did not include patients through a direct measurement of RV func- 
tion but by an indirect measure of the RV overload, as is the severity of the functional TR. However, the selection of patients by TR severity can be a confounding factor in assessing RV function [21]. The volume overload generated by TR can lead to an increase in the percentage of deformation, increasing our values. In any case, the higher predictive value of $4 \mathrm{CH}$ RV-LS over FWLS remains after including the TR severity in Cox-proportional hazard regression analysis.

A percentage of patients with coronary heart disease were included in the study and prior infarct on the right coronary artery could also reduce the RV strain, however, in our sample only 3 patients had inferior myocardial infarct and none of them extension to the RV.

The study was conducted at a single center with a relatively small sample size. The heterogeneity of the sample does not allow the analysis of absolute reference values for event prediction in each of the different etiologies.

\section{Conclusions}

The assessment of RV strain parameters is relevant for risk stratification of patients with left heart diseases. Our study shows $4 \mathrm{CH}$ RV-LS as a better predictor of heart failure episodes than the isolated determination of FWLS. Moreover, $4 \mathrm{CH}$ RV-LS provides additional prognostic information to that obtained by TAPSE and LVEF. These findings suggest that the use of $4 \mathrm{CH}$ RV-LS in clinical practice could improve the prognostic assessment of patients with left heart disease, although further studies evaluating the impact of medical decision based on the determination of the $4 \mathrm{CH}$ RV-LS on the prognosis of these patients are required.

\section{Conflict of interest: None declared}

\section{References}

1. de Groote P, Millaire A, Foucher-Hossein C et al. Right ventricular ejection fraction is an independent predictor of survival in patients with moderate heart failure. J Am Coll Cardiol, 1998; 32: 948-954.

2. Ghio S, Gavazzi A, Campana $\mathrm{C}$ et al. Independent and additive prognostic value of right ventricular systolic function and pulmonary artery pressure in patients with chronic heart failure. J Am Coll Cardiol, 2001; 37: 183-188.

3. Motoki H, Borowski AG, Shrestha K et al. Right ventricular global longitudinal strain provides prognostic value Incremental to left ventricular ejection fraction in patients with heart failure. J Am Soc Echocardiogr, 2014; 27: 726-732.

4. Mor-Avi V, Lang RM, Badano LP et al. Current and evolving echocardiographic techniques for the quantitative evaluation of cardiac mechanics: ASE/EAE consensus statement on methodol- ogy and indications endorsed by the Japanese Society of Echocardiography. Eur J Echocardiogr, 2011; 12: 167-205.

5. Haddad F, Hunt SA, Rosenthal DN, Murphy DJ. Right ventricular function in cardiovascular disease. Part I: Anatomy, physiology, aging, and functional assessment of the right ventricle. Circulation, 2008; 117: 1436-1448.

6. Kukulski T, Hübbert L, Arnold M, Wranne B, Hatle L, Sutherland GR. Normal regional right ventricular function and its change with age: A Doppler myocardial imaging study. J Am Soc Echocardiogr, 2000; 13: 194-204.

7. Ternacle J, Berry M, Cognet T et al. Prognostic value of right ventricular two-dimensional global strain in patients referred for cardiac surgery. J Am Soc Echocardiogr, 2013; 26: 721-726.

8. Simonneau G, Robbins I, Beghetti M et al. Updated clinical classification of pulmonary hypertension. J Am Coll Cardiol, 2009; 54: S43-S54.

9. Evangelista A, Flachskampf F, Lancellotti P et al. European Association of Echocardiography recommendations for standardization of performance, digital storage and reporting of echocardiographic studies. Eur J Echocardiogr, 2008; 9: 438-448.

10. Lang RM, Badano LP, Mor-Avi V et al. Recommendations for cardiac chamber quantification by echocardiography in adults: An update from the American society of echocardiography and the European association of cardiovascular imaging. Eur Heart J Cardiovasc Imaging, 2015; 16: 233-270.

11. Lancellotti P, Moura L, Pierard LA et al. European Association of Echocardiography recommendations for the assessment of valvular regurgitation. Part 2: Mitral and tricuspid regurgitation (native valve disease). Eur J Echocardiogr, 2010; 11: 307-332.

12. Meris A, Faletra F, Conca $\mathrm{C}$ et al. Timing and magnitude of regional right ventricular function: A speckle tracking-derived strain study of normal subjects and patients with right ventricular dysfunction. J Am Soc Echocardiogr, 2010; 23: 823-831.

13. Santamore WP, Dell'Italia LJ. Ventricular interdependence: Significant left ventricular contributions to right ventricular systolic function. Prog Cardiovasc Dis, 1998; 40: 289-308.

14. ParkJH,NegishiK, KwonDH, PopovicZB, GrimmRA, MarwickTH Validation of global longitudinal strain and strain rate as reliable markers of right ventricular dysfunction: Comparison with cardiac magnetic resonance and outcome. J Cardiovasc Ultrasound, 2014; 22: 113-120.

15. Cameli M, Righini FM, Lisi M, Mondillo S. Right ventricular strain as a novel approach to analyze right ventricular performance in patients with heart failure. Heart Fail Rev, 2014; 19: 603-610.

16. Iacoviello M, Forleo C, Puzzovivo A et al. Altered two-dimensional strain measures of the right ventricle in patients with Brugada syndrome and arrhythmogenic right ventricular dysplasia/ cardiomyopathy. Eur J Echocardiogr, 2011; 12: 773-781.

17. Cameli M, Lisi M, Righini FM et al. Speckle tracking echocardiography as a new technique to evaluate right ventricular function in patients with left ventricular assist device therapy. J Heart Lung Transplant, 2013; 32: 424-430.

18. Rudski LG, Lai WW, Afilalo J et al. Guidelines for the echocardiographic assessment of the right heart in adults: A report from the American Society of Echocardiography endorsed by the European Association of Echocardiography, a registered branch of the European Society of Cardiology, and the Canadian Society of Echocardiography. J Am Soc Echocardiogr, 2010; 23: 685-713.

19. Lee JH, Park JH, Park KI et al. A comparison of different techniques of two-dimensional speckle-tracking strain measurements of right ventricular systolic function in patients with acute pulmonary embolism. J Cardiovasc Ultrasound, 2014; 22: 65-71.

20. Miller D, Farah MG, Liner A, Fox K, Schluchter M, Hoit BD. The relation between quantitative right ventricular ejection fraction and indices of tricuspid annular motion and myocardial performance. J Am Soc Echocardiogr, 2004; 17: 443-447.

21. Hsiao SH, Lin SK, Wang WC, Yang SH, Gin PL, Liu CP. Severe tricuspid regurgitation shows significant impact in the relationship among peak systolic tricuspid annular velocity, tricuspid annular plane systolic excursion, and right ventricular ejection fraction. J Am Soc Echocardiogr, 2006; 19: 902-910. 